\title{
A Consideration of the Strain Amplitude-Dependent Damping and Modulus in Ferromagnetic Metals*
}

\author{
By Hisashi Kawabe** and Kaizo Kuwahara***
}

\begin{abstract}
Strain-amplitude $(\gamma)$ dependence of damping capacity $\left(Q^{-1}\right)$ and rigidity modulus $\left(f^{2}\right)$ in ferromagnetic metals (SIA and $\mathrm{Ni}$ ) has been investigated by the inverted torsion pendulum method as functions of tensile stress $\left(\sigma_{l}\right)$ and magnetic field $(H)$.

In SIA, curves of $Q^{-1}$ and $f^{2}$ vs $\gamma$ are characterized by a convex type function, which has a maximum $\left(Q_{\max }^{-1}=0.023\right)$ at the surface shear $\gamma \simeq 6 \times 10^{-5}$, and by a concave type function having a minimum $\left(f_{\min }^{2}\right)$ at nearly the same $\gamma$ as at $Q_{\max }^{-1}$, respectively. The $\gamma_{p}^{\prime}$ and $\gamma_{p}^{\prime \prime}$, which stand for the respective $\gamma$ levels at $Q_{\max }^{-1}$ and $f_{\min }^{2}$, coincide with each other at $\sigma_{l} \gtrsim 0$, whereas with increasing $\sigma_{l}$ they gradually separate making themselves larger, and for $\sigma_{l} \geqq 100 \mathrm{MPa}$, yield a rough relation, $\gamma_{p}^{\prime \prime} \simeq 2 \gamma_{p}^{\prime}$. The damping properties as a function of $H$ also have a similar tendency. On the other hand, in $\mathrm{Ni}$, the damping maximum is not observed so clearly as in SIA, and its $Q_{\max }^{-1}$ is considerably low $\left(Q_{\max }^{-1}=0.009\right.$ at $\left.\gamma_{p}^{\prime} \simeq 3 \times 10^{-4}\right)$. The variation of $\gamma_{p}^{\prime}$ with increasing $\sigma_{l}$ and $H$ leads mainly to the change in $H_{c}$ (coercive force), and therefore the difference in the damping property between $\mathrm{Ni}$ and SIA may also be ascribed to the relative magnitude of $H_{c}$.

While there has so far been given a qualitative relationship that $Q^{-1} \propto \gamma$ at $\gamma<\gamma_{p}^{\prime}$, the $Q^{-1}$ vs $\gamma\left(<\gamma_{p}^{\prime}\right)$ is more likely described by a quadratic increasing function due to the $\Delta G$-effect than by a linear increasing one, and the greater the $\Delta G$-effect, the more noticeable becomes such an increasing tendency of $Q^{-1}$.
\end{abstract}

(Received September 8, 1980)

\section{Introduction}

In recent years, so called high damping alloys $^{(1)}$ have been developed, and successful approaches to the anti-vibration by the material damping effect have been introduced accordingly ${ }^{(2)(3)}$. Among these alloys, increasing attention has been focused on ferromagnetic alloys $^{(1)}$ having the magneto-mechanical hysteresis as its damping mechanism ${ }^{(4) \sim(6)}$. The alloys are practically useful because of their high damping capacity and high rigidity irrespective of temperature or frequency, unlike the relaxation type damping alloys such as $\mathrm{Mn}-\mathrm{Cu}$ damping ones ${ }^{(7)}$.

The damping properties in ferromagnetic metals $^{(8) \sim(13)}$ have been the subject of many investigations. However, the damping mechanism needs further clarification from the

* This was originally published in Japanese in J. Japan Inst. Metals, 44 (1980), 776.

** Faculty of Engineering, The Hiroshima Institute of Technology, Itsukaichi, Hiroshima 738, Japan.

*** Faculty of Engineering, Hiroshima University, Hiroshima 730, Japan. viewpoint of necessary conditions for higher damping. In relation to the experiments of strain amplitude $(\gamma)$-dependent internal friction $\left(Q^{-1}\right)$ and elastic modulus $\left(f^{2}\right)$, it seems also that in most of the previous work, internal friction and tensile strength measurements $^{(14)(15)}$ have been carried out independently. Therefore, it is considered essential for the understanding of the damping mechanism to make clear the relation between $Q^{-1}(\gamma)$ and $f^{2}(\gamma)$ by the same vibration method under the condition of fixing domain wall movement or extinguishing domain walls.

The present paper deals with the corresponding relation between $\gamma$-dependent $Q^{-1}$ and $f^{2}$ as parameters of bias stress and applied magnetic field using wire specimens of SIA $\dagger$ and Nit†.

$\dagger$ An abbreviation of Toshiba's commertial damping metal SILENTALLOY where Fe-base alloy consisting of representative components $\mathrm{Fe}-12$ mass $\% \mathrm{Cr}-3$ mass $\% \mathrm{Al}$ is specially heat-treated to increase damping properties. It has such physical and mechanical properties as Young's modulus $\simeq$ $162 \mathrm{GPa}$, tensile strength $\simeq 426 \mathrm{MPa}$, and Vicker's hardness $\simeq 150^{(5)}$.

$\dagger \dagger \mathrm{Ni}$ wire $(\phi 1 \mathrm{~mm})$ of 99.45 mass $\%$ purity manufactured by The Japan Lamp Industrial Co. It is vacuum-annealed for $3.6 \mathrm{ks}$ at $873 \mathrm{~K}$. 
In addition, the high damping characteristics of ferromagnetic metals are discussed.

\section{Experimental Procedure}

In order to measure $\gamma$-dependent $Q^{-1}$ and $f^{2}$, an inverted torsion pendulum apparatus shown in Fig. 1 was employed. The size of specimen was $1 \mathrm{~mm}$ in diameter and $300 \mathrm{~mm}$ long. The inertia member was made into a shape of circular disk so as to prevent the occurrence of lateral vibrations introduced at the initial oscillation. Torsional displacement was derived from the variation of static capacity between the electrodes mounted on the inertia and then automatically recorded through an amplifier. At the same time, frequency $(f)$ was measured by an ultra-low frequency counter with a $10^{-3} \mathrm{~Hz}$ accuracy and automatically recorded on a printer.

The $\gamma$-dependent $Q^{-1}$ was calculated by the following formula using the $n$ - and $(n+1)$-th amplitudes $\left(A_{n}, A_{n+1}\right)$ of the measured waveform:

$$
Q^{-1}=\frac{1}{2 \pi} \cdot \frac{A_{n}^{2}-A_{n+1}^{2}}{A_{n}^{2}} .
$$

The $Q^{-1}$ and $f^{2}$ have close relations with domain wall mobility, saturated magnetostriction, etc. In order to investigate the effects of those factors on the damping properties, domain wall fixation was carried out by the methods shown in Fig. 1: (a) by tensile stress $\left(\sigma_{l}=0 \sim 280 \mathrm{MPa}\right)$, and (b) by magnetic field $(H=0 \sim 15 \mathrm{kA} / \mathrm{m})$.

\section{Results and Discussion}

\section{Strain-amplitude dependent $Q^{-1}$ and $f^{2}$ as a function of $\sigma_{l}$}

First, in Fig. 2, $\gamma$-dependent $Q^{-1}$ and $f^{2}$ are summarized as a function of $\sigma_{l}$, using the method of Fig. 1(a), where $\gamma_{m}$ is a maximum shear strain on the specimen. As for SIA, the maximum damping $\left(Q_{\max }^{-1}\right)$ becomes smaller when $\sigma_{l}$ increases, while $f^{2}$ increases with the recovery of elasticity. The highest $Q_{\max }^{-1} \simeq 0.023$ is obtained at $\gamma_{m} \simeq 6 \times 10^{-5}$ in $\sigma_{l}=0$. Also, the recovery rate of $f^{2}$ with increasing $\sigma_{l}$ is approximately $11 \%$ in this case. This convexity of $Q^{-1}$ vs $\gamma_{m}$ is peculiar to the ferromagnetic metals as observed when subjected to the stress $(\tau)$ larger than the critical stress of domain wall movement $\left(\tau_{c}\right)$. However, in consideration of the stress distribution function

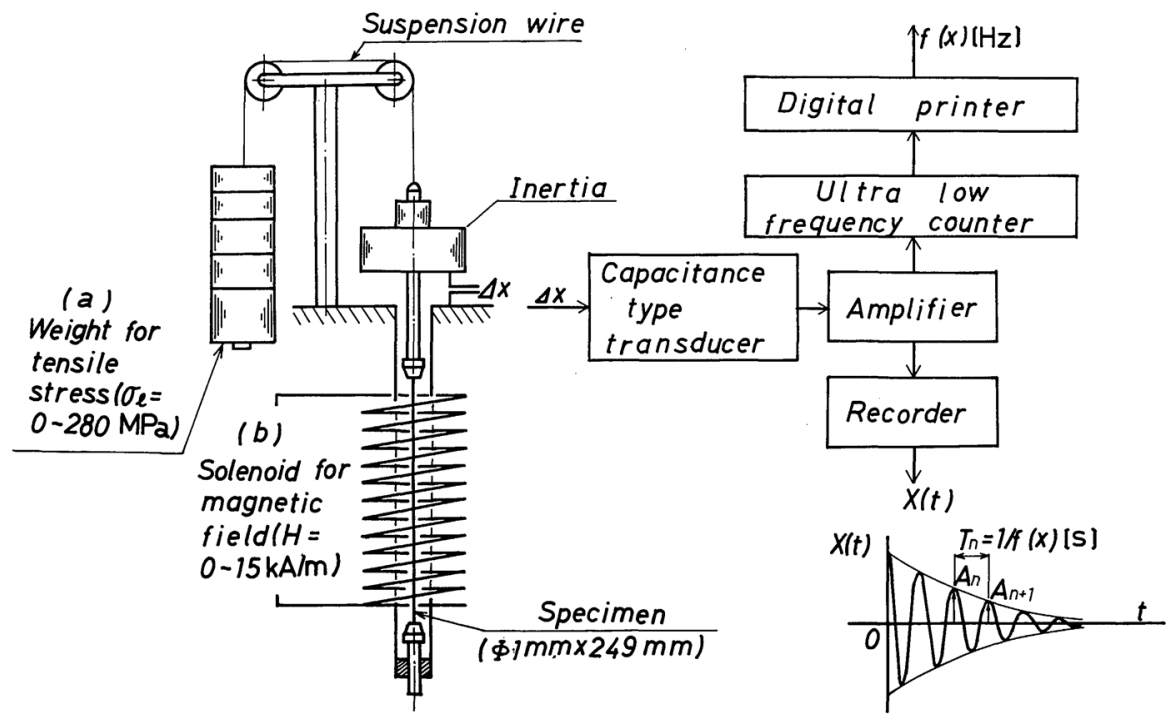

Fig. 1 Schematic illustration of the measurement system of internal friction $\left(Q^{-1}\right)$ and rigidity modulus $\left(f^{2}\right) . \Delta x$ : Small vibration displacement; $X(t)$ : recorded vibration waveform; $f(X)$ : detected frequency. 


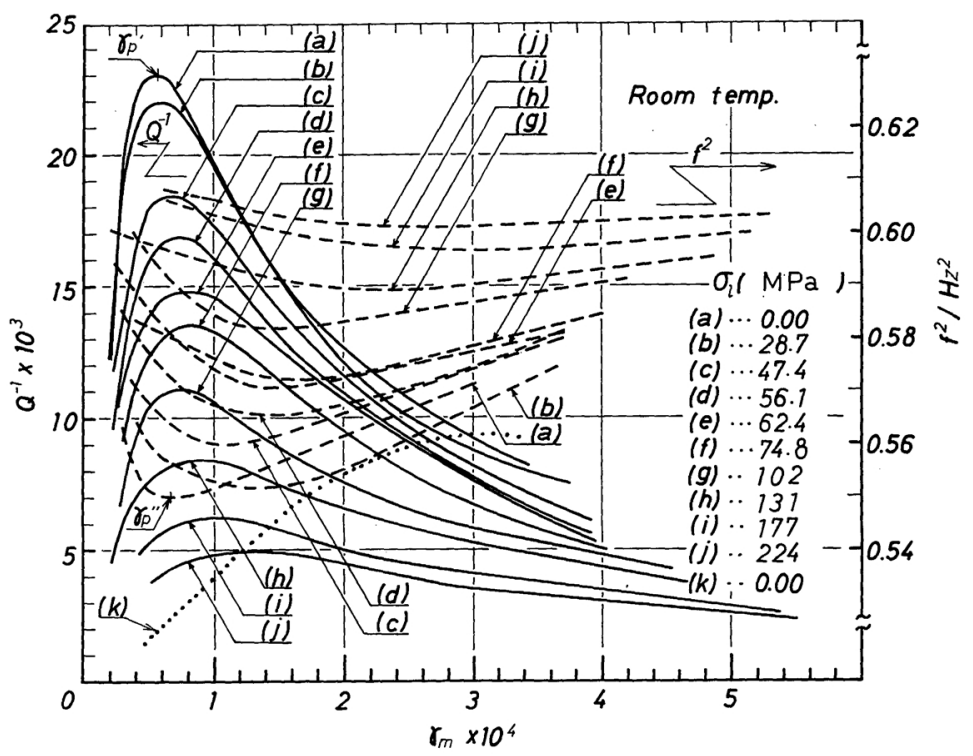

Fig. 2 Surface shear strain amplitude $\left(\gamma_{m}\right)$ dependence of $Q^{-1}$ and $f^{2}$ as a function of $\sigma_{l}$ in SIA and Ni. Curves (a) (j): $Q^{-1}$ (solid lines) and $f^{2}$ (broken lines) for SIA. Curve (k): $Q^{-1}$ for $\mathrm{Ni}$.

of the specimen (where the saturated mechanical hysteresis mechanism is dominantly operated) $\dagger$, the convex type $Q^{-1}$ characteristics may be observed more often in a wire specimen under a torsional oscillation mode rather than in a plate specimen under a transverse vibration mode.

It must be noted that as the bias stress becomes higher and the domain wall movement becomes more difficult, $\gamma_{p}^{\prime}$ and $\gamma_{p}^{\prime \prime}$ which stand for the respective $\gamma$ levels at $Q_{\max }^{-1}$ and $f_{\min }^{2}$ are gradually shifted to the higher $\gamma$ level. Figure 3 summarizes the above results. Namely, in the case of $\sigma_{l}=0, \gamma_{p}^{\prime}$ agrees with $\gamma_{p}^{\prime \prime}$, whereas, with

$\dagger$ According to Cochardt ${ }^{(16)}$, in a cantilever beam vibration system, the volume where the stress becomes $\sigma_{m}$ (maximum generated stress)/ 2 or smaller is $85 \%$, while, in a torsional oscillation system, the volume where the shear stress becomes $\tau_{m}$ (maximum shear stress) $/ 2$ or smaller is $25 \%$. In other words, in the former case, the unsaturated magneto-mechanical hysteresis mechanism operates in a large part of the beam. On the other hand, in the latter, saturated hysteresis operating volume becomes predominant. Therefore, at a higher stress than the critical one, it is easily supposed that the high stress damping by the latter may become considerably lower than that by the former.

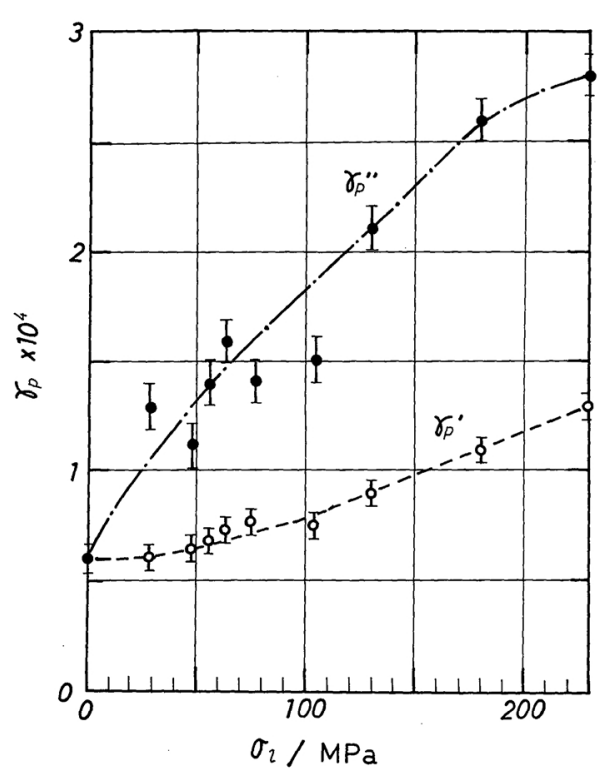

Fig. 3 Variations of the strain amplitudes $\gamma_{p}^{\prime}$ and $\gamma_{p}^{\prime \prime}$ with $\sigma_{l}$ in SIA where $Q^{-1}$ and $f^{2}$ have a maximum and a minimum, respectively.

increasing $\sigma_{l}$, they increase gradually with each other separating. In $\sigma_{l} \gtrsim 100 \mathrm{MPa}$, the following relation holds approximately:

$$
\gamma_{p}^{\prime \prime} \simeq 2 \gamma_{p}^{\prime} \text {. }
$$

It appears that those $\Delta G$ effect $^{(17)(18)}$ and the 
change in $Q^{-1}$ characteristics with increasing $\sigma_{l}$ may correspond to the change in magnetostriction and non- $180^{\circ}$ domain wall mobility. However, the coincidence of the change in $\gamma_{p}^{\prime}$ and $\gamma_{p}^{\prime \prime}$ is also observed in $\mathrm{Ni}^{(13)(19)(20)}$ though their parameters are different. In particular, the shift of $f_{\min }^{2}$ to a higher $\gamma$ level with increasing $\sigma_{l}$ agrees with the result of Honda et $a l .^{(20)}$ with respect to $\mathrm{Ni}$. The shifting trend to a higher $\gamma$ side corresponds mainly to the increase in coercive force $\left(H_{c}\right)$, as will be described later. This is considered to be a phenomenon peculiar to the ferromagnetic metals.

As shown in Fig. 2, the $Q^{-1}$ in $\mathrm{Ni}$ is also indicated. Compared with SIA, it is obvious that the maximum damping capacity is small $\left(Q_{\max }^{-1}=0.009\right)$ and the $\gamma$ giving $Q_{\max }^{-1}$ is fairly large $\left(\gamma_{p}^{\prime}=3 \times 10^{-4}\right)$. These phenomena will be discussed later.

\section{Strain-amplitude dependent $Q^{-1}$ and $f^{2}$ as a function of $\boldsymbol{H}$}

Figure 4 shows the $\gamma$-dependent $Q^{-1}$ and $f^{2}$ in SIA when the magnetic field $(H)$ is applied based on the method shown in Fig. 1. With increasing $H, Q_{\max }^{-1}$ gradually decreases, and $f_{\min }^{2}$ increases to the contrary. There is also seen close resemblance in the shift of $\gamma_{p}^{\prime}$ and $\gamma_{p}^{\prime \prime}$ to the higher $\gamma$ side between the cases of increasing $H$ and increasing $\sigma_{l}$. In addition, the $Q_{\max }^{-1}$ value for $H=0$ is slightly lower than that for

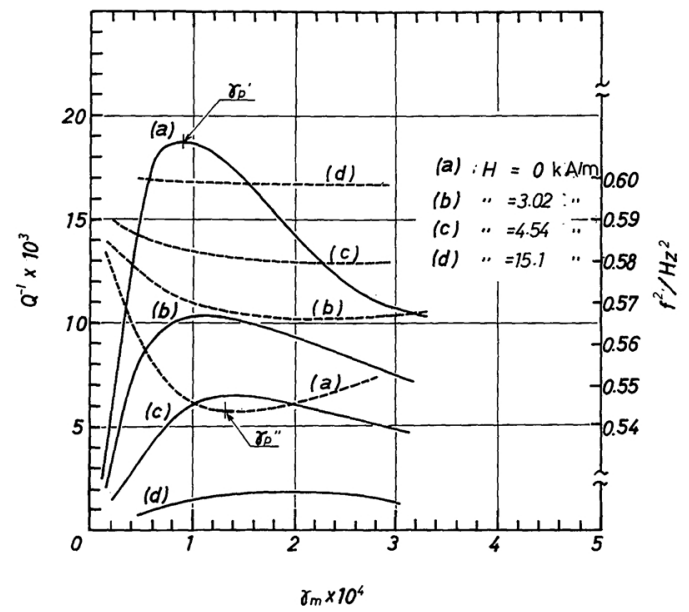

Fig. $4 Q^{-1}$ (solid lines) and $f^{2}$ (broken lines) in SIA as a function of $\boldsymbol{H}$. $\sigma_{l}=0$ (refer to Fig. 2). This is because the torsional oscillation system was previously subjected to tensile stress $\sigma_{l}=29 \sim 38 \mathrm{MPa}$ for preventing the horizontal swing generated when $H$ is applied.

Figure 5 indicates the results of the separation of $\gamma_{p}^{\prime}$ and $\gamma_{p}^{\prime \prime}$ with increasing $H$, like the case of Fig. 3. Different from the case of increasing $\sigma_{l}$, the separation of $\gamma_{p}^{\prime}$ and $\gamma_{p}^{\prime \prime}$ is already seen even in the condition of $H=0$. This seems due to the previous application of the system stabilizing tensile force to the specimen as

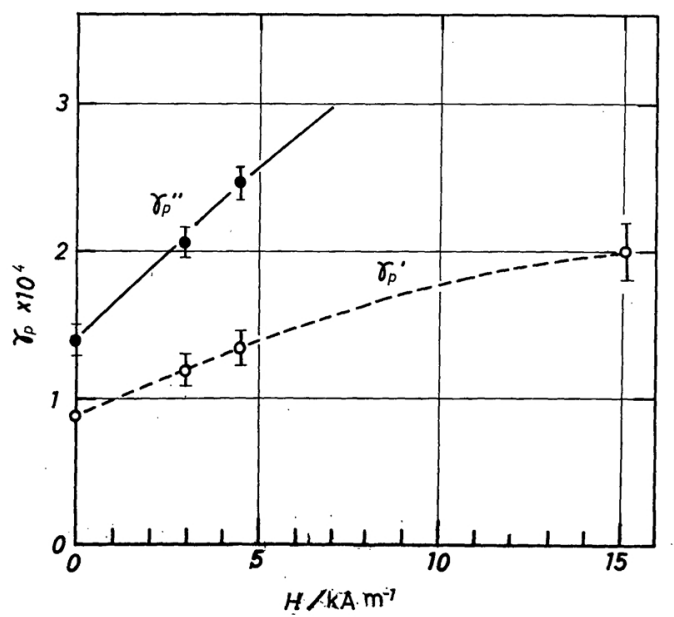

Fig. 5 Variations of $\gamma_{p}^{\prime}$ and $\gamma_{p}^{\prime \prime}$ with $H$ for SIA.

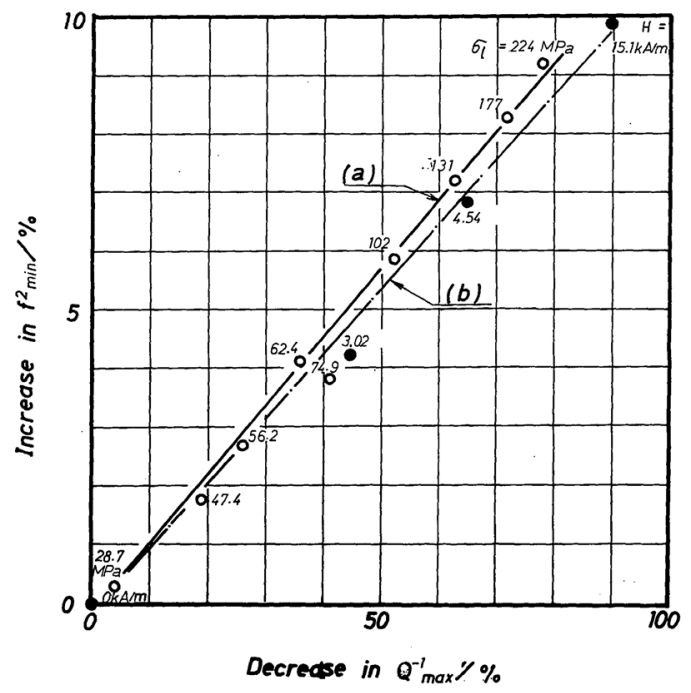

Fig. 6 Correlation between the increase in $f_{\min }^{2}$ and the decrease in $Q_{\max }^{-1}$ for SIA obtained (a) with increasing $\sigma_{l}$, and (b) with increasing $H$. 
described above. Figure 6 illustrates the correlation between $Q_{\max }^{-1}$ reduction rate and $f_{\min }^{2}$ recovery rate with increasing $\sigma_{l}$ or $H$. In either case, there is a clear linear relation between them. This implies that the damping and $\Delta G$ effect are both generated by the essentially same mechanism (i.e., change in domain wall distribution or non- $180^{\circ}$ domain wall movement due to $\sigma_{l}$ or $H$ ).

\section{Discussion of damping characteristics in terms of stress distribution condition}

Lazan $^{(21)}$ and Cochardt ${ }^{(16)(22)}$ have described the damping characteristics of materials based on the stress distribution function depending upon the material form and the loading method, and on the energy absorption function solely depending upon the material properties. In both cases, Young's modulus is assumed to be constant. It is easily deduced that as the damping capacity of ferromagnetic metal is higher, the $\Delta E$ effect ${ }^{(14)}$ becomes stronger. Accordingly, the $\gamma$-dependent elastic modulus should be especially taken into con- sideration for the analysis of the damping characteristics.

According to Rayleigh's law ${ }^{(14)}$, the energy loss $(\mathrm{d} U)$ based on the magneto-mechanical effect at low strain (i.e., the hysteresis area characterized by stress $(\tau)$-strain $(\gamma)$ ) is given by

$$
\mathrm{d} U=D \gamma_{1}^{3},
$$

where $\gamma_{1}$ is the maximum $\gamma$ of the unsaturated hysteresis characteristics (loop $l_{1}$ ), as shown in Fig. $7(b)^{(16)}$. When we express $\gamma_{c}$ as maximum $\gamma$ on the saturated hysteresis loop, i.e. critical strain, irreversible energy loss may be described approximately by the above equation in the range of $0 \leqq \gamma_{1}<\gamma_{c}$. In $\gamma$ (generated strain) $\geqq \gamma_{c}$, however, hysteresis mechanism may saturate. Therefore, if we take $\lambda$ as saturated magnetostriction, the following equation, i.e.

$$
\mathrm{d} U=K \lambda \gamma_{c},
$$

holds. Hence, from eqs. (3) and (4),

$$
D=K \lambda / \gamma_{c}^{2} \text {. }
$$

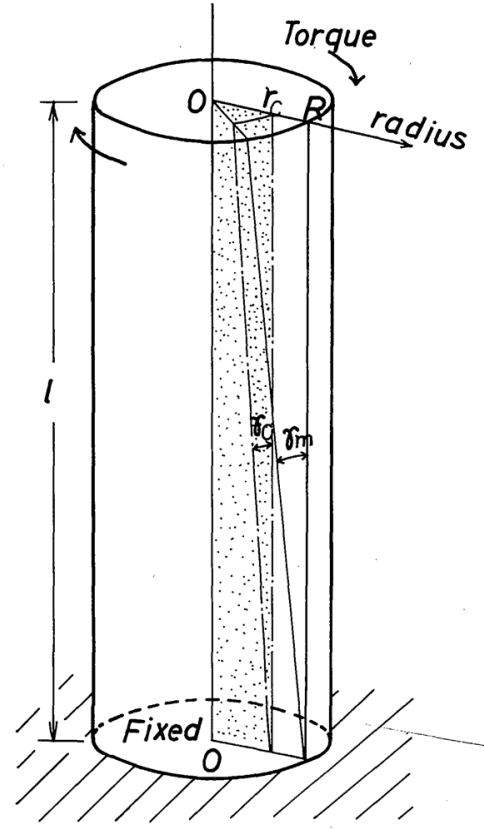

(a)

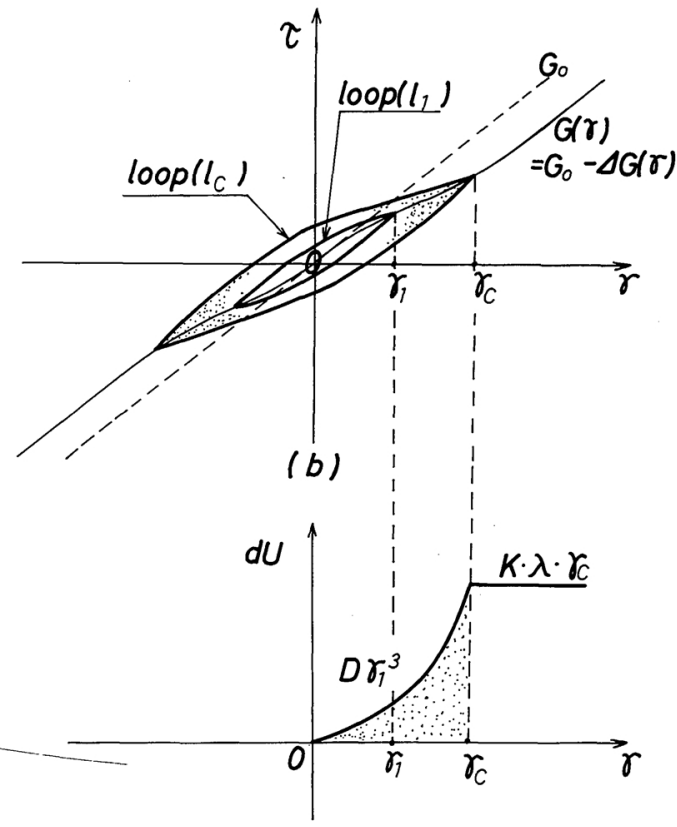

(c)

Fig. 7 (a) Strain distribution in a simple torsion bar with $\gamma_{c}$ and $\gamma_{m}$ being the critical shear. strain and the surface shear strain, respectively. (b) Mechanical hysteresis models describing unsaturated loop $\left(l_{1}\right)$ and critical hysteresis loop $\left(l_{c}\right)$. (c) Strain amplitude $(\gamma)$ dependence of the energy-dissipated function $(\mathrm{d} U)$. 
Putting $V$ and $\gamma_{m}$ as specimen volume and maximum shear strain (refer to Fig. 7(a)), respectively, the strain distribution function $^{(21)(22)}$ for the torsional mode is given by

$$
\frac{\mathrm{d} V}{\mathrm{~d} \gamma}=V \frac{2 \gamma}{\gamma_{m}^{2}}
$$

Hence, referring to eqs. (3) (6), the energy absorption function $\Delta U^{(16)}$ per unit volume and cycle is represented by

$\Delta U=\frac{1}{V} \int_{0}^{\gamma_{m}} d U \frac{\mathrm{d} V}{\mathrm{~d} \gamma} d \gamma=K \lambda \gamma_{c}\left\{1-\frac{3}{5}\left(\frac{\gamma_{c}}{\gamma_{m}}\right)^{2}\right\}$.

Then, if we describe the $\gamma$-dependent rigidity modulus by, in consideration of the $\Delta G$ effect (in this case, the $\Delta G$ effect assumes the 2 nd kind condition ${ }^{(18)}$ ),

$$
\frac{d \tau}{d \gamma}=G(\gamma)=G_{0}-\Delta G(\gamma),
$$

the mean elastic energy $U$ is given by

$$
\begin{aligned}
U & =\frac{1}{2 V} \int_{0}^{\gamma_{m}} G(\gamma) \gamma^{2} \frac{d V}{d \gamma} d \gamma \\
& =\frac{G_{0}}{\gamma_{m}^{2}} \int_{0}^{\gamma_{m}}\left\{1-\frac{\Delta G(\gamma)}{G_{0}}\right\} \gamma^{3} d \gamma .
\end{aligned}
$$

As a result, logarithmic decrement $\delta^{(21)(22)}$ considering the $\Delta G$ effect is expressed from eqs. (7) and (9) as

$$
\begin{aligned}
& \delta=\frac{\Delta U}{2 U}= \frac{2 K \lambda}{G_{0} \gamma_{c}}\left(\frac{\gamma_{c}}{\gamma_{m}}\right)^{2}\left\{1-\frac{3}{5}\left(\frac{\gamma_{c}}{\gamma_{m}}\right)^{2}\right\} \\
& \times \frac{\frac{\gamma_{m}^{4}}{4}}{\int_{0}^{\gamma_{m}}\left\{1-\frac{\Delta G(\gamma)}{G_{0}}\right\} \gamma^{3} d \gamma}, \\
&\left(: \gamma_{c} \leqq \gamma_{m}\right)
\end{aligned}
$$

where $G_{0}$ means the shear modulus under the condition that domain walls are completely fixed (or magnetically saturated condition).

The above equations are applicable to the case where $\gamma_{m}$ is sufficiently large and, therefore, critical strain $\left(\gamma_{c}\right)$ featuring the mechanical hysteresis characteristics occurs in the specimen. However, when the generated strain is considerably small and hence the hysteresis mechanism does not saturate in the specimen, the damping characteristics are given, in a similar way to eq. (10), by $\delta=\frac{4}{5} \frac{K \lambda}{G_{0} \gamma_{c}} \frac{\gamma_{m}}{\gamma_{c}} \frac{\frac{\gamma_{m}^{4}}{4}}{\int_{0}^{\gamma_{m}}\left\{1-\frac{\Delta G(\gamma)}{G_{0}}\right\} \gamma^{3} d \gamma} . \quad\left(: \gamma_{m}<\gamma_{c}\right)$

If the $G(\gamma)$ characteristics of eq. (8) are given, eqs. (10) and (11) may be analyzed. When we put $\Delta G(\gamma)=0$, eqs. (10) and (11) becomes the same as the result of Cochardt's analysis ${ }^{(16)}$. However, the originality of this treatment lies in the introduction of the $\Delta G$ effect into the damping equation, by virture of which the treatment is improved. The $G(\gamma)$ characteristics of Fig. 8(II) show the tangent modulus ${ }^{(23)}$ of the $\tau-\gamma$ characteristics ${ }^{(24)}$ in ferromagnetic metal in consideration of saturated magnetostriction $(\lambda)$ as shown in Fig. 8(I). Figure 9(b)
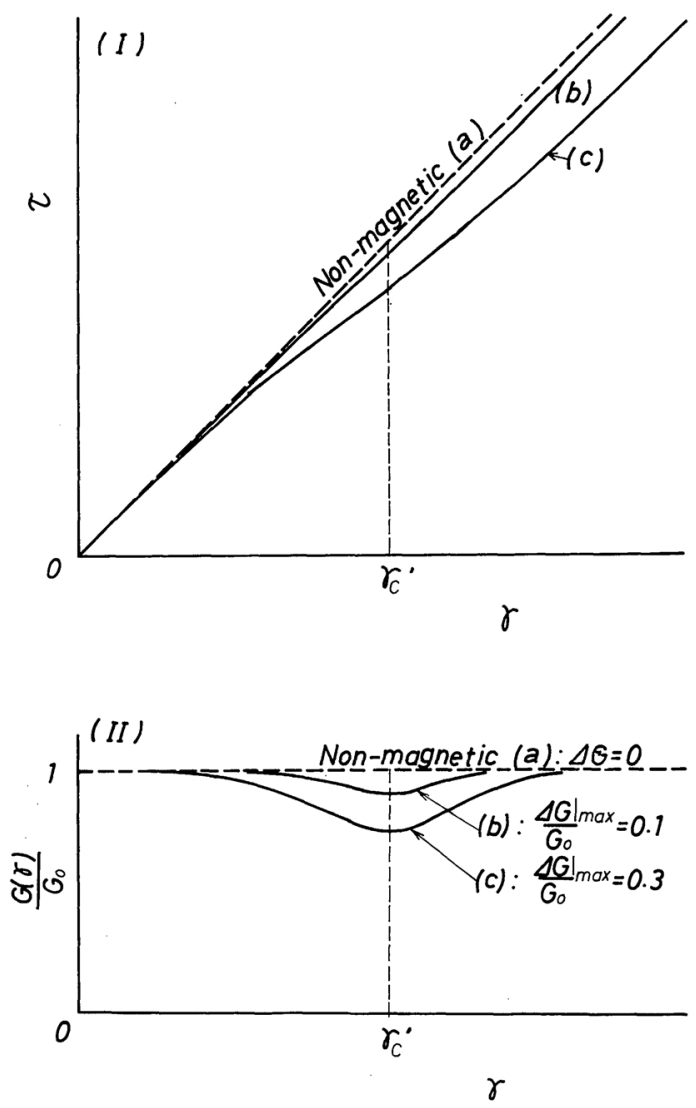

Fig. 8 (I) Schematic stress $(\tau)$-strain $(\gamma)$ curves of nonmagnetic (a) and magnetic ((b) \& (c)) materials. (II) Corresponding tangent modulus curves with $G_{0}$ and $\gamma_{c}^{\prime}$ being nonmagnetic rigidity modulus and $\gamma$ at $(d \tau / d \gamma)_{\min }$, respectively. 


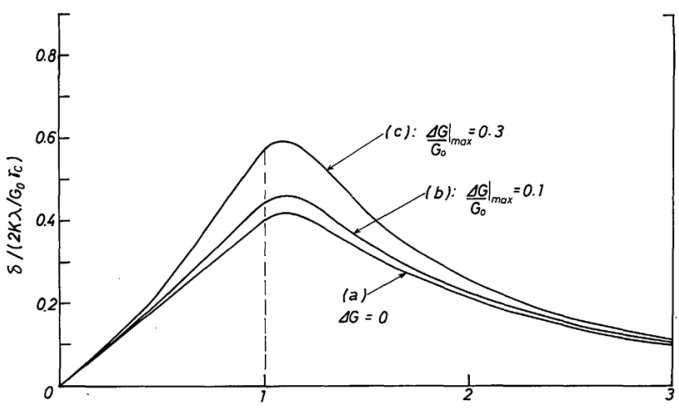

$\gamma_{m} / r_{c}$

Fig. 9 The $\gamma$-dependence of the logarithmic decrement $(\delta)$ as a function of $\Delta G / G_{0}$ under the condition of $\gamma_{c}=\gamma_{c}^{\prime}$. Curve (a): $\delta$ in the absence of the $\Delta G$-effect. Curve (b) and (c): $\delta$ in the presence of the $\Delta G$-effect.

and (c) indicate the results of numerical analysis of eqs. (10) and (11), under the condition of $\gamma_{c}=\gamma_{c}^{\prime}$, by adapting the characteristics of Fig. 8(II) (where the maximum change rate of rigidity modulus $\left(\left[\Delta G / G_{0}\right]_{\max }\right)$ due to the $\Delta G$ effect is 0.1 or 0.3$)$ to $G(\gamma)$ of eq. (8). Figure 9(a) shows the result where $\Delta G(\gamma)=0$ (i.e., the $\Delta G$ effect is neglected), indicating an approximately linear relation in a range of $\gamma_{m} / \gamma_{c}<1$.

In consideration of the $\Delta G$ effect, on the other hand, the low $\gamma$ damping characteristics may be rather featured by a quadratic increasing function than by a linear one. Moreover, the larger the $\Delta G$ effect, the more prominent becomes such a feature of $Q^{-1}$. Conventionally, the $\gamma$-dependent $Q^{-1}$ of ferromagnetic metals has qualitatively been explained to hold a relation $Q^{-1} \propto \gamma_{m}$ in the range of $\gamma_{m}<\gamma_{c}$. However, in a more strict sense, it appears to be featured by a quadratic increasing function more adequately than by a linear one, as shown by the above analysis. It also agrees with the experimental results by $\mathrm{Hagel}^{(4)}$ and Masumoto $^{(25)}$ et al. When we estimate the $Q^{-1}$ value from a damped free oscillation curve, the smaller $\gamma_{m}$, the larger the measuring error results. Also, depending on the experimental conditions of specimens (e.g. vibration frequency), there is some difference in the difficulty of the occurrence of $\Delta G$ effect $^{(18)}$. For these reasons, it seems that according to the conventional qualitative explanation, such features of the $Q^{-1}$ characteristics are apt to be disregarded. As for our experiment (refer to Fig. 2), however, a prominent quadratic increasing trend can hardly be seen upon low strain $Q^{-1}$ even in the case of $\sigma_{l}=0$. One of the reason may be the difficulty in accurate measurement of $Q^{-1}$ in the range of $\gamma \ll \gamma_{p}^{\prime}$.

The above description of the $G(\gamma)$ characteristics (Fig. 8) and the $\delta\left(\gamma_{m}\right)$ characteristics (Fig. 9) considering the $\Delta G$ effect relates originally the case where no restrictive force is acting on the movement of non- $180^{\circ}$ domain wall, i.e., the case of Fig. 2(a) experimentally (condition of $\sigma_{l}=0$ and $\gamma_{p}^{\prime} \simeq \gamma_{p}^{\prime \prime}$ ). Furthermore, the case where $\sigma_{l}$ and $H$ are operating (i.e., a case where $\gamma_{p}^{\prime}$ gradually increases and $Q_{\max }^{-1}$ decreases as shown in Figs. 2 and 4) can similarly be explained by the reduction in $\delta_{\max }$ with increasing $\gamma_{c}$, using eqs. (10) and (11) or Fig. 9. In addition, the operation of $\sigma_{l}$ and $H$ corresponds at the same time to the disappearance of the $\Delta G$ effect. Therefore, it may be considered that such a feature of $Q^{-1}$ as mentioned above (at $\gamma_{m}<\gamma_{p}^{\prime}$ ) becomes gradually ambiguous with increasing $\sigma_{l}$ or $H$. Also, the relation of eq. (2) under the stabilization of domain wall movement may be explained by replacing $\gamma_{p}^{\prime}$ and $\gamma_{p}^{\prime \prime}$ respectively with $\gamma_{c}$ and $\gamma_{c}^{\prime}$ giving $(\mathrm{d} \tau / \mathrm{d} \gamma)_{\min }$ of Fig. 9 .

Finally, the physical meaning of $\gamma_{c}$ should be discussed. From comparison of the magnetic and the mechanical hysteresis characteristics, $\gamma_{c}$ may be considered to correspond eventually to coercive force $\left(H_{c}\right)$ (although Cochard ${ }^{(22)}$ explained the correspondence between $\tau_{c}$ (critical shear stress) and $H_{c}$, those are considered essentially identical). Therefore, when $H_{c}$ is increased by the effect of stress even on the same material, $\gamma_{c}$ (in this case, equal to $\gamma_{p}^{\prime}$ ) increases as described above. At the same time, $\delta_{\max }$ has a decreasing tendency. Also, among different kinds of ferromagnetic materials, the larger $H_{c}$ of a metal, the larger becomes its $\gamma_{c}$ and, in contrast, the smaller becomes its $\delta_{\max }$ value, thus making the observation of the damping maximum difficult. Referring to Table 1 which indicates $H_{c}$ values $^{(6)(18)}$ on Ni fully annealed from $1273 \mathrm{~K}$ and SIA, and the $\gamma_{c}$ and $Q_{\max }^{-1}$ values as obtained in this experiment $\left(\sigma_{l}=0\right)$, the difference of the damping characteristics in SIA and Ni in Fig. 2 
Table 1 Values of the coercive force $\left(H_{c}\right)$, the critical shear strain $\left(\gamma_{c}\left(=\gamma_{p}^{\prime}\right)\right)$ and the $Q_{\max }^{-1}$ in SIA and $\mathrm{Ni}$.

\begin{tabular}{|c|c|c|}
\hline & SIA & $\mathrm{Ni}$ \\
\hline$H_{c}$ & $\begin{array}{l}33.4 \mathrm{~A} / \mathrm{m} \\
\left(\mathrm{Amano} \mathrm{et} \mathrm{al.}{ }^{(6)}\right)\end{array}$ & $\begin{array}{l}240 \sim 320 \mathrm{~A} / \mathrm{m} \\
\left(\text { Shirakawa et al. }{ }^{(18)}\right)\end{array}$ \\
\hline$\gamma_{c}$ & $\sim 6 \times 10^{-5}$ & $\sim 3 \times 10^{-4}$ \\
\hline$Q_{\max }^{-1}$ & 0.023 & 0.009 \\
\hline
\end{tabular}

may be explained mainly due to the relative magnitude of $H_{c}$ (i.e., difficulty in domain wall movement), though other influences can be considered, e.g. large or small saturated magnetostriction.

\section{Conclusions}

From the measurement of the strain amplitude $(\gamma)$-dependent internal friction $\left(Q^{-1}\right)$ and rigidity modulus $\left(f^{2}\right)$ in ferromagnetic metals (SIA and $\mathrm{Ni}$ ), the following results have been obtained.

(1) In SIA, there are a damping maximum $Q_{\max }^{-1}\left(Q^{-1}=0.023\right)$ and a modulus minimum $f_{\min }^{2}$ at $\gamma \simeq 6 \times 10^{-5}$, while in $\mathrm{Ni}$, such a damping peak vs $\gamma$ is not observed so clearly as in SIA $\left(Q_{\max }^{-1}=0.009\right.$ at $\left.\gamma=3 \times 10^{-4}\right)$. The difference in those damping characteristics is explained by the relative magnitude of coercive force $\left(H_{c}\right)$.

(2) When neither tensile stress $\left(\sigma_{l}\right)$ nor magnetic field $(H)$ is applied, $\gamma_{p}^{\prime}$ and $\gamma_{p}^{\prime \prime}$, which are the respective $\gamma$ levels at $Q_{\max }^{-1}$ and $f_{\min }^{2}$, coincide with each other, whereas with increasing $\sigma_{l}$ or $H$, they gradually separate making themselves larger, and yield a rough relation, $\gamma_{p}^{\prime \prime} \simeq 2 \gamma_{p}^{\prime}$ (as for SIA). Such a variation of $\gamma_{p}^{\prime}$ to higher $\gamma$ with increasing $\sigma_{l}$ or $H$ is also explained from the increase in $H_{c}$.

(3) The $\gamma$-dependent $Q^{-1}$ at $\gamma<\gamma_{p}^{\prime}$ is featured rather by a quadratic increasing function than by a linear one, where such a trend of $Q^{-1}$ is more prominent in a larger $\Delta G$ system.

\section{Acknowledgements}

The authors would like to express their gratitude to Dr. K. Sugimoto, Professor of Kansai University, for providing them with valuable data on the high damping alloys.

\section{REFERENCES}

(1) K. Sugimoto: J. Iron Steel Inst. Jap., 60 (1974), 2203.

(2) K. Sugimoto: J. Society of Automotive Engineers of Japan, 30 (1976), 154.

(3) e. g., H. Kawabe and K. Kuwahara: Bull. Jap. Soc. Prec. Engg, 14 (1980), 31.

(4) W. C. Hagel and J. M. Clark: J. Appl. Mech., Sept., (1976), 406.

(5) K. Amano, M. Sahashi, H. Tokoro and M. Nakagawa: Proc. ICIFUAS-6, Ed. by $R$. $R$. Hasiguti and N. Mikoshiba, Univ. Tokyo Press, (1977), p. 763.

(6) H. Masumoto, S. Sawaya and M. Hinai: J. Japan Inst. Metals, 41 (1977), 820 (in Japanese).

(7) K. Sugimoto, T. Mori and S. Shiode: Met. Sci. J, 7 (1973), 103.

(8) B. Becker and M. Kornetzki: Z. Phys., 88 (1934), 634.

(9) W. Cooke: Phys. Rev., 50 (1963), 1158.

(10) H. Franz: Z. Mettallk., 53 (1962), 27.

(11) J. A. Roberts and P. Barrand: Acta Met., 17 (1969), 757.

(12) A. W. Cochardt: J. Appl. Phys., 25 (1954), 670.

(13) Y. Tanji, Y. Shirakawa and H. Moriya: J. Japan Inst. Metals, 33 (1969), 1354 (in Japanese).

(14) R. M. Bozorth: Ferromagnetism, D. Van Nostrand Co., New York, (1965), p. 685.

(15) K. Amano, S. Sato and H. Noji: Synopsis of the 1977 Spring Meeting of Jap. Inst. Metals, (1977), p. 162.

(16) A. W. Cochardt: J. Appl. Mech., Sept., 21 (1954), 257.

(17) H. Saito and Y. Tanji: Bulletin Japan Inst. Metals: 10 (1971), 436 (in Japanese).

(18) U. Shirakawa and Y. Tanji: J. Japan Inst. Metals, 33 (1969), 819 (in Japanese).

(19) A. V. Nest et A. Dubé: Comt. rend., 255 (1962), 1732.

(20) K. Honda and T. Terada: Phil. Mag., 13 (1907), 36.

(21) B. J. Lazan: J. Appl. Mech., June, 20 (1953), 201.

(22) A. W. Cochardt: J. Appl. Mech., June, 20 (1953), 196.

(23) B. J. Lazan: Damping of Materials and Members in Structural Mechanics, Pergamon Press, (1968), p. 100.

(24) H. Franz: Z. Metallk., 53 (1962), 27.

(25) H. Masumoto, S. Sawaya and M. Hinai: J. Japan Inst. Metals, 43 (1979), 472 (in Japanese). 